\title{
Trapping of Oxonium Ylide with Isatins: Efficient and \\ Stereoselective Construction of Adjacent Quaternary \\ Carbon Centers
}

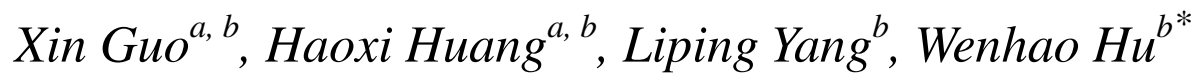

${ }^{a}$ Chengdu Institute of Organic Chemistry and Chinese Academy of

Sciences and Graduate School of Chinese Academy of Sciences,

China

${ }^{b}$ Department of chemistry, East China Normal University, Shanghai 200062, China

\section{Supporting Information}

\section{General}

All reactions were performed under an argon atmosphere in a well-dried reaction flask. Melting points are uncorrected. ${ }^{1} \mathrm{H}$ and ${ }^{13} \mathrm{CNMR}$ spectra were recorded at 300 $\mathrm{MHz}$ and $75 \mathrm{MHz}$ with Brucker-300 MHz spectrometer. HRMS (ESI) Mass spectra were recorded on BRUKER FT-MS. Dichloromethane was distilled over calcium hydride.

2. General Procedure for three-component reaction of diazo compounds with alcohols and isatins

To a refluxing $\mathrm{CH}_{2} \mathrm{Cl}_{2}(5 \mathrm{~mL})$ solution of $\mathrm{Rh}_{2}(\mathrm{OAc})_{4}(2.25 \mathrm{mg}, 1 \% \mathrm{~mol})$, benzyl alcohol $\mathbf{2 a}(65 \mu \mathrm{L}, 0.60 \mathrm{mmol})$ and isatin $\mathbf{3 a}(80 \mathrm{mg}, 0.50 \mathrm{mmol})$ under argon atmosphere was added methyl phenyldiazoacetate 1a (106mg, 0.60mmol) in $2 \mathrm{~mL}$ of $\mathrm{CH}_{2} \mathrm{Cl}_{2}$ over 2 $\mathrm{h}$ via a syringe pump. After the addition was completed, the reaction mixture was cooled to room temperature. Solvent was removed, and the crude product was purified by flash column chromatography on silica gel eluting with $25 \%$ EtOAc-light petroleum to give $\mathbf{4 a}(192 \mathrm{mg}), 93 \%$ yield. Products $\mathbf{4 b}-\mathbf{4 p}$ were obtained by the same procedure. 
Methyl 2-(benzyloxy)-2-(3-hydroxy-1-methyl-2-oxoindolin-3-yl)-2-phenylacetate (4a)

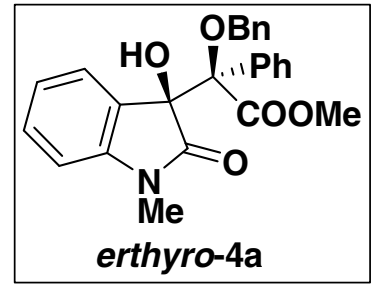

Yield, 93\%; TLC $\mathrm{R}_{\mathrm{f}}=0.40$ (petroleum: acetate $=3: 2$ );

${ }^{1} \mathrm{HNMR}\left(\mathrm{CDCl}_{3}, 300 \mathrm{MHz}\right): \delta 2.85(\mathrm{~s}, 3 \mathrm{H}), 3.92(\mathrm{~s}, 3 \mathrm{H}), 4.60(\mathrm{dd}$, $\mathrm{J}=11.4 \mathrm{~Hz}), 5.84(\mathrm{~s}, 1 \mathrm{H}), 6.35(\mathrm{~d}, \mathrm{~J}=7.8 \mathrm{~Hz}), 6.97(\mathrm{~m}, 1 \mathrm{H})$, 7.05-7.16(m, 6H), 7.37-7.50(m, 6H) ${ }^{13} \mathrm{CNMR}\left(\mathrm{CDCl}_{3}, 75\right.$ $\mathrm{MHz}): \delta 25.56,52.86,68.08,81.64,86.36,107.47,122.60$, $125.80,126.49,126.70,127.16,127.74,128.09,128.47,128.77$, 129.70, 130.08, 132.42, 137.45, 143.32, 171.01, 175.20;HRMS: calcd for $\mathrm{C}_{25} \mathrm{H}_{23} \mathrm{NNaO}_{5}[\mathrm{M}+\mathrm{Na}]^{+}, 440.1474$; found 440.1486

Methyl 2-(benzyloxy)-(3-hydroxy-1-phenyl-2-oxoindolin-3-yl)-2-phenylacetate (4b)

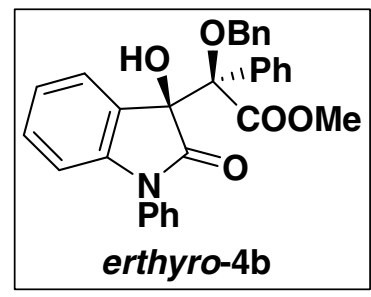

Yield, 93\%; TLC Rf $=0.50$ (petroleum: acetate $=3: 2$ ); ${ }^{1} \mathrm{HNMR}\left(\mathrm{CDCl}_{3}, 300 \mathrm{MHz}\right): \delta 3.92(\mathrm{~s}, 3 \mathrm{H}), 4.59-4.70(\mathrm{~m}, 2 \mathrm{H})$, $6.10(\mathrm{~s}, 1 \mathrm{H}), 6.34-6.37(\mathrm{~d}, \mathrm{~J}=7.5 \mathrm{~Hz}, 1 \mathrm{H}), 7.01-7.52(\mathrm{~m}, 14 \mathrm{H})$. ${ }^{13} \mathrm{CNMR}\left(\mathrm{CDCl}_{3}, 75 \mathrm{MHz}\right): \delta 52.88,68.07,81.73,86.33$, $108.80,123.03,126.06,126.51,126.87,127.02,127.19,127.46$, $127.75,128.00,128.15,128.45,129.10,129.36,129.58,129.88$, $132.46,133.49,137.33,143.33,170.98,174.60$

HRMS: calcd for $\mathrm{C}_{30} \mathrm{H}_{25} \mathrm{NNaO}_{5}[\mathrm{M}+\mathrm{Na}]^{+}, 502.1630$; found 502.1625

Methyl 2-(benzyloxy)-2-(3-hydroxy-2-oxoindolin-3-yl)-2-phenylacetate (4c)

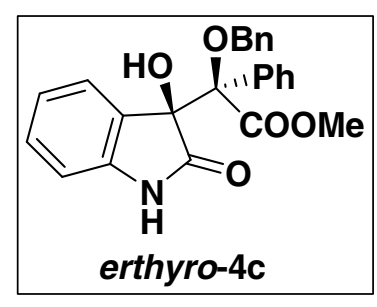

Yield, 90\%; TLC Rf $=0.25$ (petroleum: acetate $=3: 2$ );

${ }^{1} \mathrm{HNMR}\left(\mathrm{CDCl}_{3}, 300 \mathrm{MHz}\right): \delta 3.87(\mathrm{~s}, 3 \mathrm{H}), 4.64(\mathrm{~s}, 2 \mathrm{H}), 5.82(\mathrm{~s}$, $1 \mathrm{H}), 6.48(\mathrm{~d}, \mathrm{~J}=7.7 \mathrm{~Hz}, 1 \mathrm{H}), 6.88-6.89(\mathrm{~m}, 1 \mathrm{H}), 7.09-7.49(\mathrm{~m}$, $12 \mathrm{H}), 7.78(\mathrm{~s}, 1 \mathrm{H}) ;{ }^{13} \mathrm{CNMR}\left(\mathrm{CDCl}_{3}, 75 \mathrm{MHz}\right): \delta 52.81, \quad 68.21$, $81.57,86.31,109.39,122.50,126.96,127.09,127.16,127.72$, $128.27,128.46,128.98,129.74,132.37,137.52,140.63,171.06$, 171.22; HRMS: calcd for $\mathrm{C}_{24} \mathrm{H}_{21} \mathrm{NO}_{5}[\mathrm{M}+\mathrm{Na}]^{+}, 426.1317$; found 426.1312

Methyl 2-(benzyloxy)-2-(3-hydroxy-5-nitro-2-oxoindolin-3-yl)-2-phenylacetate (4d)

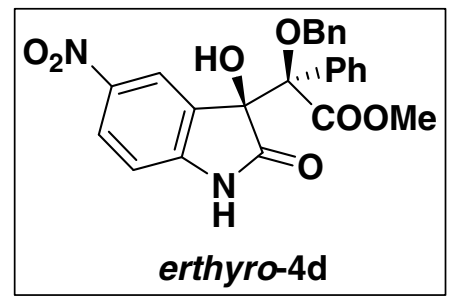

Yield, $81 \%$; TLC Rf $=0.30$ (petroleum: acetate $=1: 1$ );

${ }^{1} \mathrm{HNMR}\left(\mathrm{CDCl}_{3}, 300 \mathrm{MHz}\right): \quad 83.88(\mathrm{~s}, 3 \mathrm{H}), \quad 4.69(\mathrm{~s}, 3 \mathrm{H})$, $5.87(\mathrm{~s}, 1 \mathrm{H}), 6.61(\mathrm{~d}, \mathrm{~J}=8.6 \mathrm{~Hz}, 1 \mathrm{H}), 7.18-7.27(\mathrm{~m}, 5 \mathrm{H})$, 7.35-7.48(m, 5H), 7.60(s, 1H), 8.04-8.09(m, 2H); ${ }^{13} \mathrm{CNMR}(\mathrm{DMSO}, 75 \mathrm{MHz}): \delta 176.401,169.412,149.081$, $141.388,138.329,133.152,130.072,128.911,128.497$, $128.231,127.311,126.791,126.619,121.842,109.291,87.299,79.527,67.805$, 52.360; HRMS: calcd for $\mathrm{C}_{24} \mathrm{H}_{20} \mathrm{~N}_{2} \mathrm{NaO}_{7}[\mathrm{M}+\mathrm{Na}]^{+}, 471.1168$; found 471.1163 
Methyl 2-(benzyloxy)-2-(5-chloro-3-hydroxy-2-oxoindolin-3-yl)-2-phenylacetate (4e)

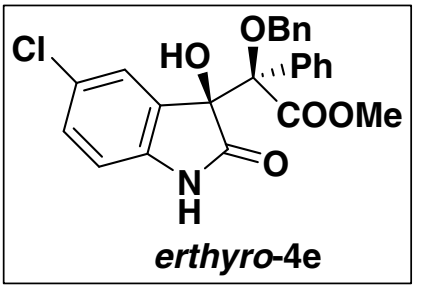

Yield, 85\%; TLC Rf $=0.40$ (petroleum: acetate $=1: 1$ );

${ }^{1} \mathrm{HNMR}\left(\mathrm{CDCl}_{3}, 300 \mathrm{MHz}\right): \quad \delta 7.45(\mathrm{~m}, 4 \mathrm{H}), \quad 7.37(\mathrm{~s}, 1 \mathrm{H})$, $7.26(\mathrm{~m}, 7 \mathrm{H}), 7.08-7.05(\mathrm{~d}, \mathrm{~J}=9 \mathrm{~Hz}, 1 \mathrm{H}), 6.43-6.40(\mathrm{~d}, \mathrm{~J}=9 \mathrm{~Hz}$, $1 \mathrm{H}), 5.85(\mathrm{~s}, 1 \mathrm{H}), 4.64(\mathrm{~s}, 2 \mathrm{H}), 3.86(\mathrm{~s}, 3 \mathrm{H}) ;{ }^{13} \mathrm{CNMR}:\left(\mathrm{CDCl}_{3}\right.$, $75 \mathrm{MHz}): \delta 176.582,170.898,139.102,137.316,132.102$, $129.686,128.927,128.549,127.849,127.582,127.351$,

127.121, 110.207, 86.115, 81.493, 68.420, 52.912; HRMS: calcd for $\mathrm{C}_{24} \mathrm{H}_{20} \mathrm{ClNNaO}_{5}[\mathrm{M}+\mathrm{Na}]^{+}, 460.0928$; found 460.0922

Methyl 2-(benzyloxy)-2-(5-bromo-3-hydroxy-2-oxoindolin-3-yl)-2-phenylacetate (4f)

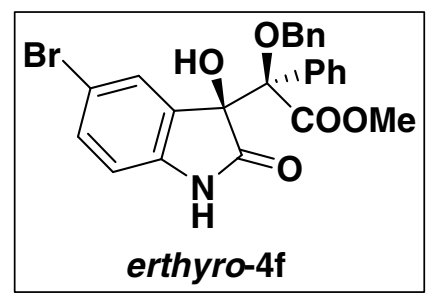

Yield, 82\%; TLC Rf=0.45 (petroleum: acetate $=1: 1$ );

${ }^{1} \mathrm{HNMR}\left(\mathrm{CDCl}_{3}, \quad 300 \mathrm{MHz}\right): \quad \delta 7.47-7.30(\mathrm{~m}, \quad 7 \mathrm{H})$, $7.23-7.17(\mathrm{~m}, 6 \mathrm{H}), 6.38-6.35(\mathrm{~d}, 1 \mathrm{H}, \mathrm{J}=8.4 \mathrm{~Hz}), 5.83(\mathrm{~s}, 1 \mathrm{H})$, 4.65-4.64(m, 2H) , 3.86(s, 3H); ${ }^{13} \mathrm{CNMR}\left(\mathrm{CDCl}_{3}, 75 \mathrm{MHz}\right)$ : $\delta 176.402,170.889,139.598,137.332,132.572,132.110$, $130.407,129.225,128.927,128.561,127.843,127.367$,

127.110, 115.103, 110.661, 86.140, 81.424, 68.445, 52.909; HRMS: calcd for $\mathrm{C}_{24} \mathrm{H}_{20} \mathrm{BrNNaO}_{5}[\mathrm{M}+\mathrm{Na}]^{+}, 504.0423$; found 504.0417

Methyl 2-(3-hydroxy-1-methyl -2-oxoindolin-3-yl)-2-methoxy-2-phenylacetate (4h)

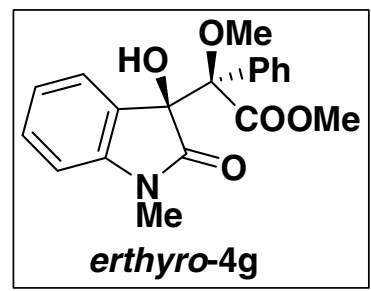

Yield, 79\%; TLC Rf=0.35 (petroleum: acetate $=3: 2$ );

${ }^{1} \mathrm{HNMR}\left(\mathrm{CDCl}_{3}, \quad 300 \mathrm{MHz}\right): \quad \delta 7.48-7.45(\mathrm{~d}, \quad \mathrm{~J}=7.3 \mathrm{~Hz}, \quad 1 \mathrm{H})$, 7.13-7.04(m, 6H), 6.99-6.93(t, $1 \mathrm{H}), 6.36-6.33(\mathrm{~d}, \quad \mathrm{~J}=7.7 \mathrm{~Hz}$, $1 \mathrm{H}), 3.97(\mathrm{~s}, 3 \mathrm{H}), 3.43(\mathrm{~s}, 3 \mathrm{H}), 2.84(\mathrm{~s}, 3 \mathrm{H}) ;{ }^{13} \mathrm{CNMR}\left(\mathrm{CDCl}_{3}\right.$, $75 \mathrm{MHz}): \delta 175.118,170.873,143.240,131.984,132.102$, $129.755,128.763,127.974,126.631,126.344,122.612$, 107.531, 86.661, 81.409, 54.195, 52.882, 25.575; HRMS: calcd for $\mathrm{C}_{19} \mathrm{H}_{19} \mathrm{NNaO}_{5}[\mathrm{M}$ $+\mathrm{Na}]^{+}, 364.1161$; found 364.1155

Methyl 2-(3-hydroxy-1-methyl -2-oxoindolin-3-yl)-2-phenyl-2-isopropoxyacetate (4i)

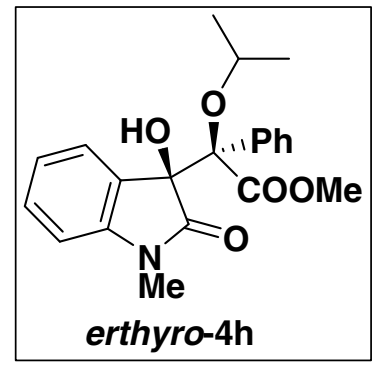

Yield, 81\%; TLC Rf=0.40 (petroleum: acetate $=3: 2$ );

${ }^{1} \mathrm{HNMR}\left(\mathrm{CDCl}_{3}, \quad 300 \mathrm{MHz}\right): \quad \delta 7.62-7.60(\mathrm{~d}, \quad \mathrm{~J}=6.8 \mathrm{~Hz}, \quad 1 \mathrm{H})$, $7.15-6.97(\mathrm{~m}, 7 \mathrm{H}), 6.31-6.29(\mathrm{~d}, \mathrm{~J}=7.7 \mathrm{~Hz}, 1 \mathrm{H}), 6.20(\mathrm{~s}, 1 \mathrm{H})$, $3.98(\mathrm{~s}, \quad 4 \mathrm{H}), \quad 2.75(\mathrm{~s}, \quad 3 \mathrm{H}), \quad 1.31-1.29(\mathrm{~d}, \quad \mathrm{~J}=6.1 \mathrm{~Hz}, \quad 3 \mathrm{H})$, $1.25-1.23(\mathrm{~d}, \mathrm{~J}=6 \mathrm{~Hz}, 3 \mathrm{H}) ;{ }^{13} \mathrm{CNMR}\left(\mathrm{CDCl}_{3}, 75 \mathrm{MHz}\right): \delta 175.377$, $172.090,143.232,133.679,129.617,129.084,127.849$, $127.781,126.807,126.114,112.458,107.352,85.414,81.762$, 69.629, 52.835, 25.403, 24.305, 22.719; HRMS: calcd for $\mathrm{C}_{21} \mathrm{H}_{23} \mathrm{NNaO}_{5}[\mathrm{M}+\mathrm{Na}]^{+}$, 392.1474 ; found 392.1468 


\section{Methyl}

\section{2-tert-butoxy-2-(3-hydroxy-1-methyl-2-oxoindolin-3-yl)-2-phenylacetate $(4 \mathbf{j})$}

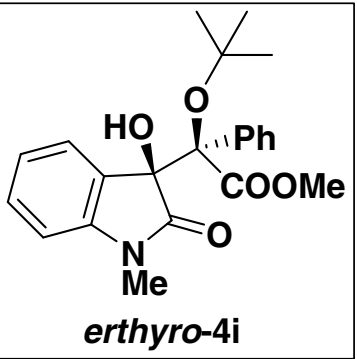

Yield, 72\%; TLC Rf=0.50 (petroleum: acetate $=3: 2$ ); ${ }^{1} \mathrm{HNMR}\left(\mathrm{CDCl}_{3}, \quad 300 \mathrm{MHz}\right): \quad \delta 7.68-7.65(\mathrm{~d}, \quad \mathrm{~J}=7.2 \mathrm{~Hz}, \quad 1 \mathrm{H})$, 7.17-7.07(m, 3H), 7.00-6.90(m, 4H), 6.26-6.24(m, 2H), 4.01(s, $3 \mathrm{H}), 2.72(\mathrm{~s}, 3 \mathrm{H}), 1.33(\mathrm{~s}, 9 \mathrm{H}) ;{ }^{13} \mathrm{CNMR}\left(\mathrm{CDCl}_{3}, 75 \mathrm{MHz}\right)$ : $\delta 175.550,172.576,143.053,135.394,129.462,129.107$, $127.849,127.345,126.570,125.458,122.408,107.246,83.546$ $82.447,79.359,52.835,29.948,25.343$; HRMS: calcd for

$\mathrm{C}_{22} \mathrm{H}_{25} \mathrm{NNaO}_{5}[\mathrm{M}+\mathrm{Na}]^{+}, 406.1630$; found 406.1625

Methyl 2-hydroxy-2-(3-hydroxy-1-methyl-2-oxoindolin-3-yl)-2-phenylacetate(4g) (mixture of diastereomers)

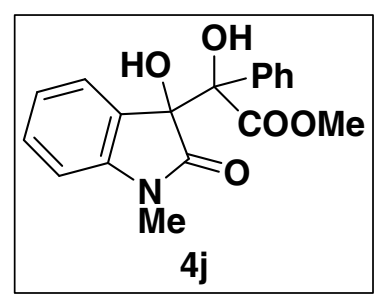

Yield, 96\%; TLC Rf $=0.25$ (petroleum: acetate $=3: 2$ );

${ }^{1} \mathrm{HNMR}\left(\mathrm{CDCl}_{3}, \quad 300 \mathrm{MHz}\right): \quad \delta 7.78-7.76(\mathrm{~d}, \quad \mathrm{~J}=6.0 \mathrm{~Hz}, \quad 2 \mathrm{H})$, 7.60-7.31(m, 4H), 6.84-6.75(t, 2H), 5.98-5.95(d, J=7.0Hz, $1 \mathrm{H}), \quad 4.92(\mathrm{~s}, 1 \mathrm{H}), 4.01(\mathrm{~s}, 3 \mathrm{H}), 3.81(\mathrm{~s}, 1 \mathrm{H}), 3.18(\mathrm{~s}, 3 \mathrm{H})$; ${ }^{13} \mathrm{CNMR}\left(\mathrm{CDCl}_{3}, 75 \mathrm{MHz}\right): \delta 176.35,173.25,14482,135.72$, $134.96,130.68,130.10,129.77,128.856,128.74,127.77$,

$127.46,127.14,125.82,125.69,125.02,123.10,122.91,122.57,122.35,108.40$, 108.16, 79.92, 77.94, 69.76, 54.103, 53.09, 52.77, 29.64, 26.11; HRMS: calcd for $\mathrm{C}_{18} \mathrm{H}_{17} \mathrm{NNaO}_{5}[\mathrm{M}+\mathrm{Na}]^{+}, 350.1004$; found 350.0999

\section{Methyl}

2-(benzyloxy)-2-(3-hydroxy-1-methyl-2-oxoindolin-3-yl)-2-(4-methoxyphenyl) acetate $(4 \mathbf{k})$

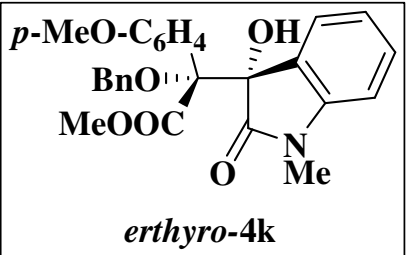

Yield, 80\%; TLC Rf=0.45 (petroleum: acetate=3:2); ${ }^{1} \mathrm{HNMR}\left(\mathrm{CDCl}_{3}, 300 \mathrm{MHz}\right): \delta 7.48-7.38(\mathrm{~m}, 6 \mathrm{H}), 7.12-7.05(\mathrm{~m}$, $3 \mathrm{H}), 6.93-6.92(\mathrm{t}, 1 \mathrm{H}), 6.61-6.57(\mathrm{~d}, 2 \mathrm{H}, \mathrm{J}=9 \mathrm{~Hz}), 6.41-6.38(\mathrm{~d}$, $1 \mathrm{H}, \mathrm{J}=7.8 \mathrm{~Hz}), 5.84(\mathrm{~s}, 1 \mathrm{H}), 4.61-4.57(\mathrm{~m}, 2 \mathrm{H}), 3.91(\mathrm{~s}, 3 \mathrm{H})$, $3.70(\mathrm{~s}, 3 \mathrm{H}), 2.87(\mathrm{~s}, 3 \mathrm{H}) ;{ }^{13} \mathrm{CNMR}\left(\mathrm{CDCl}_{3}, 75 \mathrm{MHz}\right)$ :

$\delta 175.246,171.192,159.077,143.300,137.439,130.110,129.633,128.390,127.641$, 127.066, 126.764, 126.306, 124.027, 122.530, 112.047, 107.585, 85.94, 81.51, 67.793, 55.039, 52.807, 25.573; HRMS: calcd for $\mathrm{C}_{26} \mathrm{H}_{25} \mathrm{NNaO}_{6}[\mathrm{M}+\mathrm{Na}]^{+}, 470.1580$; found 470.1574 


\section{Methyl}

\section{2-(benzyloxy)-2-(4-chlorophenyl)-2-(3-hydroxy-1-methyl-2-oxoindolin-3-yl) acetate (4l)}

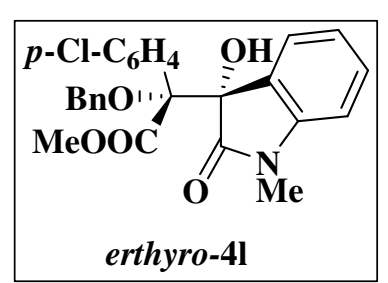

Yield, 84\%; TLC Rf=0.35 (petroleum: acetate=3:2); ${ }^{1} \mathrm{HNMR}\left(\mathrm{CDCl}_{3}, 300 \mathrm{MHz}\right): 87.50-7.37(\mathrm{~m}, 6 \mathrm{H}), 7.14-6.94(\mathrm{~m}$, $6 \mathrm{H}), 6.42-6.40(\mathrm{~d}, \mathrm{~J}=8.4 \mathrm{~Hz}, 1 \mathrm{H}), 5.81(\mathrm{~s}, 1 \mathrm{H}), 4.54(\mathrm{~s}, 2 \mathrm{H})$, $3.93(\mathrm{~s}, 3 \mathrm{H}), 2.88(\mathrm{~s}, 3 \mathrm{H}) ;{ }^{13} \mathrm{CNMR}\left(\mathrm{CDCl}_{3}, 75 \mathrm{MHz}\right): \delta 174.989$, $170.565,143.171,137.076,134.116,131.096,130.248$, $129.970,128.524,127.883,127.141,126.884,126.393$,

122.796, 107.778, 85.958, 81.493, 68.109, 53.058, 29.660, 25.640; HRMS: calcd for $\mathrm{C}_{25} \mathrm{H}_{22} \mathrm{ClNNaO}_{5}[\mathrm{M}+\mathrm{Na}]^{+}, 474.1084$; found 474.1079

Methyl 2-(benzyloxy)-2-(3-chlorophenyl)-2-(3-hydroxy-1-methyl-2-oxoindolin-3-yl) acetate (4m)

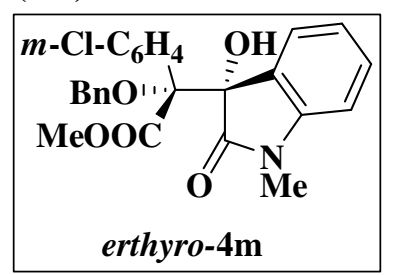

Yield, 82\%; TLC Rf=0.35 (petroleum: acetate $=3: 2$ );

${ }^{1} \mathrm{HNMR}\left(\mathrm{CDCl}_{3}, 300 \mathrm{MHz}\right): \delta 7.49-7.40(\mathrm{~m}, 6 \mathrm{H}), 7.18-7.10(\mathrm{~m}, 3 \mathrm{H})$, 7.08-7.01(m, 3H), 6.41-6.38(d, J=7.8Hz, 1H), 5.76(s, 1H), 4.56(m, 2H), 3.94(s, 3H), 2.90(s, 3H); ${ }^{13} \mathrm{CNMR}\left(\mathrm{CDCl}_{3}, 75 \mathrm{MHz}\right): \delta 174.877$, $170.322,143.059,136.970,134.716,132.884,130.274,129.946$,

$129.047,128.725,128.517,128.140,127.903,127.802,127.240,126.903,126.356,126.257$, 125.893, 122.803, 107.670, 86.026, 81.526, 68.215, 53.088, 29.633, 25.579; HRMS: calcd for $\mathrm{C}_{25} \mathrm{H}_{22} \mathrm{NO} 5[\mathrm{M}+\mathrm{Na}]^{+}, 474.1084$; found 474.1079

Methyl 2-(benzyloxy)-2-(3-chlorophenyl)-2-(3-hydroxy-1-methyl-2-oxoindolin-3-yl) acetate (4n)

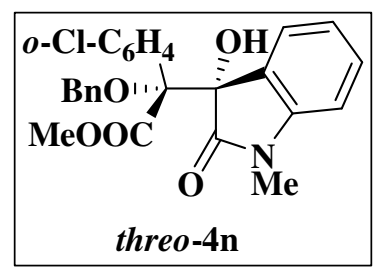

Yield, 24\%; TLC Rf=0.35 (petroleum: acetate=3:2);

${ }^{1} \mathrm{HNMR}\left(\mathrm{CDCl}_{3}, 300 \mathrm{MHz}\right): \delta 7.47-7.27(\mathrm{~m}, 7 \mathrm{H}), 7.25-7.10(\mathrm{~m}, 4 \mathrm{H})$, 6.82-6.80(t, $1 \mathrm{H}), 6.78(\mathrm{~s}, 1 \mathrm{H}), 6.55-6.53(\mathrm{~d}, \mathrm{~J}=7.8 \mathrm{~Hz}, 1 \mathrm{H}), 5.02-4.98(\mathrm{~d}$, $\mathrm{J}=11.8 \mathrm{~Hz}, 1 \mathrm{H}), 4.72-4.68(\mathrm{~d}, \mathrm{~J}=11.8 \mathrm{~Hz}, 1 \mathrm{H}), 3.67(\mathrm{~s}, 3 \mathrm{H}), 3.05(\mathrm{~s}, 3 \mathrm{H})$;

${ }^{13} \mathrm{CNMR}\left(\mathrm{CDCl}_{3}, 75 \mathrm{MHz}\right): \delta 175.67,168.92,144.50,137.90,133.44$, $132.82,131.39,130.27,130.04,129.72,128.51,127.67,127.19,126.59$, 125.89, 125.71, 121.92, 107.44, 85.56, 69.578, 52.46, 29.34, 27.07; HRMS: calcd for $\mathrm{C}_{25} \mathrm{H}_{22} \mathrm{NNaO} 5[\mathrm{M}+\mathrm{Na}]^{+}, 474.1084$; found 474.1079

Ethyl 2-(benzyloxy)-2-(3-hydroxy-1-methyl-2-oxoindolin-3-yl) acetate (4o) (mixture of diastereomers)

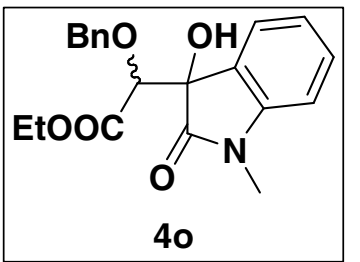

Yield, 89\%; TLC $\mathrm{Rf}=0.40 \quad$ (petroleum: acetate $=3: 2$ ); ${ }^{1} \mathrm{HNMR}\left(\mathrm{CDCl}_{3}, 300 \mathrm{MHz}\right): \delta 7.35-7.20(\mathrm{~m}, 15 \mathrm{H}), 7.08-7.03(\mathrm{~m}$, $2 \mathrm{H}), 6.81-6.78(\mathrm{~d}, \mathrm{~J}=7.8 \mathrm{~Hz}, 2 \mathrm{H}), 4.87-4.83(\mathrm{~d}, \mathrm{~J}=11.4 \mathrm{~Hz}, 1 \mathrm{H})$, 4.70-4.67(d, J=11.7Hz, $1 \mathrm{H}), 4.50(\mathrm{~s}, 1 \mathrm{H}), 4.46(\mathrm{~s}, 1 \mathrm{H}), 4.42(\mathrm{~s}$, $1 \mathrm{H}), 4.33(\mathrm{~s}, 1 \mathrm{H}), 4.30-4.20(\mathrm{~m}, 2 \mathrm{H}), 4.13-4.08(\mathrm{~m}, 2 \mathrm{H}), 3.18(\mathrm{~s}$,

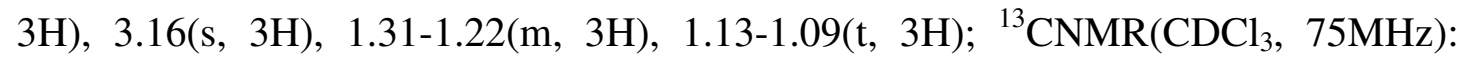
$\delta 175.81,175.43,171.40,170.41,145.21,137.45,131.20,131.07,129.58,129.50$, $129.33,129.16,129.00,128.90,128.45,127.81,125.83,125.54,123.91,123.87$, 
109.30, 109.10, 81.12, 80.74, 75.15, 74.70, 68.74, 68.09, 62.63, 62.47, 27.18, 27.15, 14.99, 14.78; HRMS: calcd for $\mathrm{C}_{20} \mathrm{H}_{21} \mathrm{NNaO}_{5}[\mathrm{M}+\mathrm{Na}]^{+}, 378.1317$; found 378.1312

\section{3-(1-(Benzyloxy)-2-oxo-2-phenylethyl)-3-hydroxy-1-methylindolin-2-one}

(4r)(mixture of diastereomers)

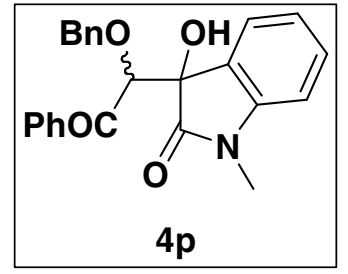

Yield, 88\%; TLC $\mathrm{Rf}=0.45$ (petroleum: acetate $=3: 2$ ); ${ }^{1} \mathrm{HNMR}\left(\mathrm{CDCl}_{3}, \quad 300 \mathrm{MHz}\right): \quad \delta 7.91-7.88(\mathrm{~d}, \quad \mathrm{~J}=9 \mathrm{~Hz}, \quad 2 \mathrm{H})$, 7.65-7.62(d, J=8.9Hz, 2H), 7.60-7.45(m, 2H), 7.40-7.28(m, 2H), 7.26-7.21(m, 2H), 7.19-7.02(m, 2H), 6.78-6.75(d, J=9Hz, $1 \mathrm{H})$, $6.69-6.66(\mathrm{~d}, \mathrm{~J}=8.9 \mathrm{~Hz}, 1 \mathrm{H}), 5.18(\mathrm{~s}, 1 \mathrm{H}), 5.14(\mathrm{~s}, 1 \mathrm{H}), 4.80-4.76(\mathrm{~d}$, $\mathrm{J}=11.1 \mathrm{~Hz}, 1 \mathrm{H}), 4.62-4.58(\mathrm{~d}, \mathrm{~J}=10 \mathrm{~Hz}, 1 \mathrm{H}), 4.58-4.54(\mathrm{~d}, \mathrm{~J}=11.1 \mathrm{~Hz}, 1 \mathrm{H}), 4.44-4.40(\mathrm{~d}$, $\mathrm{J}=10 \mathrm{~Hz}, 1 \mathrm{H}), 4.25(\mathrm{~s}, 1 \mathrm{H}), 4.09(\mathrm{~s}, 1 \mathrm{H}), 3.12(\mathrm{~s}, 1 \mathrm{H}), 2.85(\mathrm{~s}, 1 \mathrm{H}) ;{ }^{13} \mathrm{CNMR}\left(\mathrm{CDCl}_{3}\right.$, 75MHz): $\delta 199.14,198.24,175.67,174.67,144.11,143.23,136.55,133.47,133.13$, $130.00,129.04,128.63,128.41,128.28,128.19,128.12,128.07,127.97,127.87$, $125.23,123.01,122.76,108.32,108.13,84.23,82.17,74.08,73.06,26.17,25.87$; HRMS: calcd for $\mathrm{C}_{24} \mathrm{H}_{21} \mathrm{NNaO}_{4}[\mathrm{M}+\mathrm{Na}]^{+}, 410.1368$; found 410.1363

\section{Crystal data}

$\lambda=.71073 \AA$, the structure was solved by direct methods (SHELXL-97) and refined by full-matrix least-squares on $\mathrm{F}^{2}$. Compound $\mathbf{4 d}: \mathrm{C}_{24} \mathrm{H}_{20} \mathrm{~N}_{2} \mathrm{O}_{7}, \mathrm{M}=448.42$, monoclinic, space group P2(1)/n, a = 10.085 (1) $\AA, b=16.279(2) \AA, c=13.421(2) \AA, \alpha=90^{\circ}$, $\beta=101.56(1)^{\circ}, \gamma=90^{\circ}, V=2158.7(5) \AA^{3}, Z=4, \mu=0.103 \mathrm{~mm}^{-1}, \mathrm{~T}=287(2) \mathrm{K}, 4853$ reflections collected, 4015 unique $(\mathrm{Rint}=0.0125)$. Final $\mathrm{GooF}=1.004, \mathrm{R}^{1}=0.0397$, $\mathrm{wR}^{2}=0.0997, \mathrm{R}$ indices based on 4015 reflections with $\mathrm{I}>2 \sigma(\mathrm{I}), 308$ parameters, 0 restraints, $\mathrm{CCDC}$

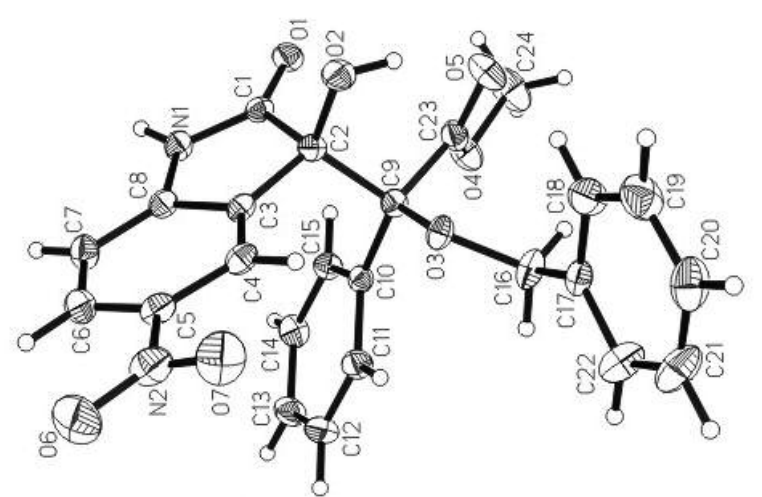

Figure 1. ORTEP representation of the crystal structure of erthyro-4d 


\section{General procedures for rhodium-catalyzed three-component reaction in supercritical $\mathrm{CO}_{2}$}

A cylindrical stainless steel reactor was charged with $\mathrm{Rh}_{2}(\mathrm{OAc})_{4}(4.42 \mathrm{mg}$, $0.01 \mathrm{mmol})$, benzyl alcohol $(125 \mu \mathrm{L}, 1.2 \mathrm{mmol})$ and istain $\mathbf{3 a}(193 \mathrm{mg}, 1 \mathrm{mmol})$, followed by pressurization with $\mathrm{CO}_{2}(15.2 \mathrm{MPa})$ with cooling in an ice-bath. Upon warming the reactor to $40^{\circ} \mathrm{C}$, a supercritical phase was produced $\left(19.4 \mathrm{MPa}\right.$ total pressure, $\left.40^{\circ} \mathrm{C}\right)$. Liquid methyl phenyldiazoacetate $(177 \mathrm{mg}, 1.0 \mathrm{mmol})$ was then pumped into the reactor, and the resulting $\mathrm{scCO}_{2}$ phase $\left(19.8 \mathrm{MPa}\right.$ pressure, $\left.40^{\circ} \mathrm{C}\right)$ was stirred for $10 \mathrm{~min}$. By releasing the pressure, $\mathrm{scCO}_{2}$ was easily removed, leaving behind the crude product as a yellow solid. The pure product was isolated in 95\% yield (396mg) with 99:1 diastereoseletivity. 